TRANSACTIONS OF THE

AMERICAN MATHEMATICAL SOCIETY

Volume 353, Number 12, Pages 4949-4962

S 0002-9947(01)02749-0

Article electronically published on April 18, 2001

\title{
THE CURVE OF "PRYM CANONICAL" GAUSS DIVISORS ON A PRYM THETA DIVISOR
}

\author{
ROY SMITH AND ROBERT VARLEY
}

\begin{abstract}
The Gauss linear system on the theta divisor of the Jacobian of a nonhyperelliptic curve has two striking properties:

1) the branch divisor of the Gauss map on the theta divisor is dual to the canonical model of the curve;

2) those divisors in the Gauss system parametrized by the canonical curve are reducible.

In contrast, Beauville and Debarre prove on a general Prym theta divisor of dimension $\geq 3$ all Gauss divisors are irreducible and normal. One is led to ask whether properties 1) and 2) may characterize the Gauss system of the theta divisor of a Jacobian. Since for a Prym theta divisor, the most distinguished curve in the Gauss system is the Prym canonical curve, the natural analog of the canonical curve for a Jacobian, in the present paper we analyze whether the analogs of properties 1) or 2) can ever hold for the Prym canonical curve. We note that both those properties would imply that the general Prym canonical Gauss divisor would be nonnormal. Then we find an explicit geometric model for the Prym canonical Gauss divisors and prove the following results using Beauville's singularities criterion for special subvarieties of Prym varieties:
\end{abstract}

Theorem. For all smooth doubly covered nonhyperelliptic curves of genus $g \geq 5$, the general Prym canonical Gauss divisor is normal and irreducible.

Corollary. For all smooth doubly covered nonhyperelliptic curves of genus $g \geq 4$, the Prym canonical curve is not dual to the branch divisor of the Gauss map.

\section{INTRODUCTION}

A good understanding of the geometry of a theta divisor $\Theta$ of a principally polarized abelian variety $(A, \Theta)$ requires a knowledge of properties of its canonical linear system, the Gauss linear system $\left|\mathcal{O}_{\Theta}(\Theta)\right|$. A striking feature of the theta divisor $\Theta(C)$ of the Jacobian of a curve $C$ is that the dual of the branch divisor of the associated Gauss map $\gamma_{\Theta}$ on $\Theta$, is not a hypersurface as expected but a nondegenerate curve $\left[\mathrm{A}\right.$, namely the canonical model $C_{\omega} \subset\left|\omega_{C}\right|^{*} \cong\left|\mathcal{O}_{\Theta}(\Theta)\right|$ of $C$. This feature is so striking that one is led to ask whether it is shared by other principally polarized abelian varieties, for example by those p.p.a.v.'s most similar to Jacobians, the Prym varieties. For the Prym variety $(P, \Xi)$ of a connected étale double cover of curves $\pi: \tilde{C} \rightarrow C$ the most natural first question of this type is whether the branch divisor of $\gamma_{\Xi}$ is dual to the Prym canonical model $\varphi_{\eta}(C)=C_{\eta} \subset\left|\omega_{C} \otimes \eta\right|^{*}$ of the curve $C$. Specialization to a Jacobian (an approach

Received by the editors January 4, 1999 and, in revised form, April 15, 2000.

2000 Mathematics Subject Classification. Primary 14Hxx, 14Kxx. 
proposed in private communication by Donagi), e.g. by letting $C$ become trigonal, seems to imply that the set of Prym varieties whose Gauss map $\gamma_{\Xi}$ has branch divisor dual to $C_{\eta}$ is a union of proper subvarieties of all Pryms, but gives no information on the possible number of such subvarieties. One obstacle to proving a more precise result has been a lack of computable models for the divisors in the Gauss linear system on a Prym theta divisor.

In the present paper we construct explicit models for the Gauss divisors $\Gamma_{\bar{p}}$ parametrized by points $\varphi_{\eta}(\bar{p})$ of the Prym canonical curve $C_{\eta}$ and deduce from their geometry that in fact $C_{\eta}$ is never dual to a component of the branch divisor of $\gamma_{\Xi}$, for any connected étale double cover $\tilde{C} \rightarrow C$ of any nonhyperelliptic curve $C$ of genus $g \geq 4$. (These may be considered as limiting cases of the models $S_{p q}$ given in [BD2, proof of Prop. 1, p.615] for proper intersections of translates of theta divisors on Prym varieties.) Although this means one cannot repeat for any Prym varieties Andreotti's proof [A] of the Torelli theorem, it raises the question of whether the property that the dual of the branch divisor of the Gauss map is a nondegenerate curve may be characteristic of Jacobians. This question remains open even among Prym varieties. A result of Beauville and Debarre stated in BD2, Remarque 1, p. 619], along with a result in the present paper, seems to imply that the set of Prym varieties of dimension $\geq 4$ whose Gauss map $\gamma_{\Xi}$ has branch divisor dual to any curve is a union of proper subvarieties of all Pryms, and that Jacobians are an irreducible component of this union, but again leaves open the possible number of such subvarieties. An alternate model for the Gauss map on a Prym theta divisor given by Verra in $[\overline{\mathrm{Ve}}]$, although complicated, has enabled him to compute the degree of $\gamma_{\Xi}$ for a generic Prym, and could eventually be useful in determining the branch divisor. For generic four-dimensional Pryms, i.e. all generic p.p.a.v.'s of dimension four, Adams, McCrory, Shifrin, and Varley have computed in unpublished work AMSV1, AMSV2, that both the branch divisor of $\gamma_{\Xi}$ and its dual variety, are irreducible surfaces of degree 60 in $\mathbb{P}^{3}$. Since for a general $\operatorname{Prym}(P, \Xi)$ (of dimension $\geq 8), C_{\eta}$ does equal the base locus of the tangent cones at double points of $\Xi$, the present result exhibits another contrast between theta divisors of Pryms and theta divisors of Jacobians of curves (of genus $\geq 5$ ) and of intermediate Jacobians of cubic threefolds, where the dual of the branch divisor of $\gamma_{\Theta}$ is equal, in general, to the base locus of the tangent cones at singular points.

The method of constructing the divisors $\Gamma_{\bar{p}}$ in this paper is the following: the Prym theta divisor $\Xi$ associated to a double cover $\tilde{C} \rightarrow C$ with genus $(C)=g$, admits a surjective map $\varphi: X \rightarrow \Xi$ with generic fiber $\mathbb{P}^{1}$, where $X \subset \tilde{C}^{(2 g-2)}$ parametrizes certain divisors on $\tilde{C}$ of degree $2 g-2$ (see "The setup" below for precise definitions). Each point $p$ in $\tilde{C}$ determines a generic section $\mathcal{D}_{p} \subset X$ of this map in the sense that if $\mathcal{D}_{p}=$ those divisors $D$ in $X$ such that $p$ belongs to $D\}$, then the restriction $\varphi: \mathcal{D}_{p} \rightarrow \Xi$ has degree one. Since each $\mathcal{D}_{p}$ thus maps birationally to $\Xi$, for any $p \neq q$ on $\tilde{C}$ the image $\varphi\left(\mathcal{D}_{p} \cap \mathcal{D}_{q}\right) \subset \Xi$ should represent a "self-intersection" of $\Xi$ and hence is a candidate for an element of the Gauss linear system $\left|\mathcal{O}_{\Xi}(\Xi)\right|$. The cases $q \neq p, p^{\prime}$ are computed in [BD2, p.615], and are not Gauss divisors, but proper intersections of translates of $\Xi$. The limiting case $q=p^{\prime}$, is a Gauss divisor, which we compute as follows.

Theorem. If $p$ and $p^{\prime}$ are the preimages of a point $\bar{p}$ of $C$ via the étale connected double cover $\tilde{C} \rightarrow C$, where $C$ is smooth and nonhyperelliptic of genus $g \geq 4$, then 
1) $\varphi\left(\mathcal{D}_{p} \cap \mathcal{D}_{p^{\prime}}\right)=\Gamma_{\bar{p}} \subset \Xi$ is the Gauss divisor corresponding to the point $\varphi_{\eta}(\bar{p})$ on $C_{\eta} \subset\left|\omega_{C} \otimes \eta\right|^{*} \cong\left|\mathcal{O}_{\Xi}(\Xi)\right|$.

2) If furthermore $g \geq 5$ and $\bar{p}$ is a general point of $C$, then $\bar{p}$ is a ramification point of only finitely many $g_{4}^{1}$ 's, and for any such $\bar{p}, \Gamma_{\bar{p}}$ is normal and irreducible.

3) If the curve $C_{\eta}$ were dual to a component of the branch divisor of $\gamma_{\Xi}$ on $\Xi_{s m}$, then each $\Gamma_{\bar{p}}$ would be singular in codimension one.

It follows (with a special argument for genus 4) that $C_{\eta}$ is never dual to a component of the branch divisor of $\gamma_{\Xi}$ on $\Xi_{s m}$, for any étale connected double cover $\tilde{C} \rightarrow C$ of any smooth nonhyperelliptic curve $C$ of genus $g \geq 4$. We sketch the proof of the main result: the theta divisor $\Xi$ of the Prym variety of an étale double cover $\pi: \tilde{C} \rightarrow C$ of a curve $C$ of genus $g$, can be modeled by "precanonical effective even line bundles on $\tilde{C}$ ", i.e. $\Xi=\left\{\xi \in \operatorname{Pic}^{2 g-2}(\tilde{C}): h^{0}(\xi) \geq 2\right.$ and even, and $\left.\mathrm{N}(\xi)=\omega_{C}\right\}$ where $\mathrm{N}$ is the norm map $\mathrm{N}: \operatorname{Pic}(\tilde{C}) \rightarrow \operatorname{Pic}(C)$. Consequently, the Abel map $\tilde{\alpha}: \tilde{C}^{(2 g-2)} \rightarrow \tilde{\Theta}$ restricts to a surjective map $\varphi: X \rightarrow \Xi$ with generic fiber $\mathbb{P}^{1}$, where $X=\tilde{\alpha}^{-1}(\Xi)$. One can then relate the structure of $X$ and $\varphi$ to the Gauss linear system on $\Xi$ as follows: for a double cover $\pi: \tilde{C} \rightarrow C$ if $p \in \tilde{C}$, and $X \supset \mathcal{D}_{p}=\{$ precanonical effective even divisors containing $p\}$, then for every $p \neq q$ in $\tilde{C}$, Beauville's homological calculations in $\left[\mathrm{B}\right.$ imply that $\Xi \supset \varphi\left(\mathcal{D}_{p} \cap \mathcal{D}_{q}\right)$ has the homology class $[\Xi]^{2}$ of a Gauss divisor on $\Xi$. We then complete the proof of part 1) of the theorem by proving a set theoretic inclusion $\varphi\left(\mathcal{D}_{p} \cap \mathcal{D}_{p^{\prime}}\right) \subset \Gamma_{\bar{p}}$, where $\bar{p}$ is any point of $C$ and $\left\{p, p^{\prime}\right\}=\pi^{-1}(\bar{p})$.

The organization of the paper is as follows: after setting up the definitions, conventions, and notations, we restate the main theorem and its corollary. We prove the corollary first, assuming the theorem, followed by a proof of each of the three parts of the theorem in order. At the end of the paper we make some remarks on the case when the base curve $C$ of the double cover is hyperelliptic, the relation with Andreotti's result, and more detailed remarks on related results of other workers including those above. Finally, we pose some open questions, for example: if a component of the branch divisor of the Gauss map on an indecomposable principally polarized abelian variety $(A, \Theta)$ is dual to a nondegenerate curve, is $(A, \Theta)$ a Jacobian?

The setup. We work over the complex numbers. If $C$ is a smooth connected projective curve of genus $g \geq 2, \operatorname{Pic}(C)$ its Picard variety, and $\operatorname{Pic}^{2 g-2}(C) \supset$ $\Theta=\left\{L: h^{0}(L) \neq 0\right\}$ the natural theta divisor, the pair $\left(\operatorname{Pic}^{0}(C),[\Theta]\right)$, where $[\Theta]$ is the homology class of any translate of $\Theta$ in $\operatorname{Pic}^{0}(C)$, is the Jacobian of $C$, a principally polarized abelian variety denoted $J(C)$. If $\pi: \tilde{C} \rightarrow C$ is a connected étale double cover, and $N: \operatorname{Pic}(\tilde{C}) \rightarrow \operatorname{Pic}(C)$ the associated norm map on line bundles, then $N^{-1}(0)=\left\{L \in \operatorname{Pic}^{0}(\tilde{C}): N(L)=\mathcal{O}_{C}\right\}$ has two connected components, and the Prym variety of $\pi$ is by definition [Mu1, p.331] $P^{0}=$ the $(g-1)$ dimensional connected component of $N^{-1}(0)$ which contains 0 , with principal polarization (equal to half that) induced from $\operatorname{Pic}^{0}(\tilde{C})$. We consider also Mu1 p.342] the associated "precanonical" cosets of the Prym variety, both the "odd" one $P^{-}=N^{-1}\left(\omega_{C}\right)^{\text {odd }}=\left\{L: N(L)=\omega_{C}, h^{0}(L)\right.$ odd $\} \subset \operatorname{Pic}^{2 g-2}(\tilde{C})$, and the "even" one $P^{+}=P=N^{-1}\left(\omega_{C}\right)^{\text {ev }}=\left\{L: N(L)=\omega_{C}, h^{0}(L)\right.$ even $\} \subset \operatorname{Pic}^{2 g-2}(\tilde{C})$, the latter with natural divisor $\Xi=\Xi(\tilde{C} / C)=(P \cap \tilde{\Theta})_{\text {red }}=$ the "Prym theta divisor", and 
its parametrization $\varphi: X \rightarrow \Xi$ (with fiber $\cong \mathbb{P}^{1}$ over a generic point of any component of $\Xi$ ) defined by the restriction of the Abel map $\tilde{\alpha}: \tilde{C}^{(2 g-2)} \rightarrow \operatorname{Pic}^{2 g-2}(\tilde{C})$ to $\tilde{\alpha}^{-1}(\Xi)=X . P^{-}$admits a parametrization $\varphi^{-}: X^{-} \rightarrow P^{-}$where $\varphi^{-}$is a map with fiber $\cong \mathbb{P}^{0}$ over a generic point of $P^{-}$, and $\varphi^{-}$is defined as the restriction of the Abel map $\tilde{\alpha}: \tilde{C}^{(2 g-2)} \rightarrow \operatorname{Pic}^{2 g-2}(\tilde{C})$ to $\tilde{\alpha}^{-1}\left(P^{-}\right)=X^{-}$. Furthermore, when $C$ is nonhyperelliptic, [B, Cor. of Prop. 3, p.365] $X$ and $X^{-}$are irreducible. We should remark that in $[\mathrm{B}]$ the definition of the scheme structure on $X, X^{-}$is different from that given above, i.e. if $\alpha: C^{(2 g-2)} \rightarrow \mathrm{Pic}^{2 g-2}(C)$ is the Abel map for $C, \pi: \tilde{C}^{(2 g-2)} \rightarrow C^{(2 g-2)}$ the norm map on divisors, and $\left\{\omega_{C}\right\}$ the canonical point in $\mathrm{Pic}^{2 g-2}(C)$, then in $[\mathrm{B}$, p.359, line -6$]$ the scheme structures on $X, X^{-}$ are defined as in [W, (8.3), p.99], i.e. as inherited from that defined by the equation $X \cup X^{-}=\pi^{-1}\left(\left|\omega_{C}\right|\right)$. But in fact the two scheme structures $\pi^{-1}\left(\left|\omega_{C}\right|\right)$ and $\tilde{\alpha}^{-1}\left(\Xi \cup P^{-}\right)$on $X \cup X^{-}$agree, first of all since $\pi^{-1}\left(\left|\omega_{C}\right|\right)=(\alpha \circ \pi)^{-1}\left(\omega_{C}\right)=$ $(N \circ \tilde{\alpha})^{-1}\left(\omega_{C}\right)=\tilde{\alpha}^{-1}\left(P \cup P^{-}\right)$. Then since the scheme $\tilde{\alpha}^{-1}\left(P \cup P^{-}\right)$is reduced by [B, p.359], and contains the scheme $\tilde{\alpha}^{-1}\left(\Xi \cup P^{-}\right)$, and since as sets $\tilde{\alpha}^{-1}\left(P \cup P^{-}\right)$ and $\tilde{\alpha}^{-1}\left(\Xi \cup P^{-}\right)$are equal, then as schemes they are equal as well.

If $p$ is a point of $\tilde{C}$, and $\mathcal{D}_{p} \subset X$ is the subset of $X$ whose corresponding divisors on $\tilde{C}$ contain $p$, then we claim $\mathcal{D}_{p} \subset X$ is a divisor with nonempty intersection with every fiber of $\varphi$, hence $\varphi\left(\mathcal{D}_{p}\right)=\Xi$. I.e. since the finite norm map $\pi: \tilde{C}^{(2 g-2)} \rightarrow$ $C^{(2 g-2)}$, maps $X$ onto $\left|\omega_{C}\right|$ and $\mathcal{D}_{p} \subset X$ onto the codimension one subspace $\mid \omega_{C}-$ $\bar{p}|+\bar{p} \subset| \omega_{C} \mid, \mathcal{D}_{p}$ is a divisor in $X$. Since $\mathcal{D}_{p}$ meets each positive dimensional linear series $\varphi^{-1}(y)$ in codimension at most one, $\mathcal{D}_{p} \cap \varphi^{-1}(y) \neq \emptyset$ for every $y$ in $\Xi$.

Our main observation is that when $C$ is nonhyperelliptic of genus $g \geq 4$, then the set $\varphi\left(\mathcal{D}_{p} \cap \mathcal{D}_{p^{\prime}}\right)$ with its reduced scheme structure is the Prym canonical Gauss divisor $\Gamma_{\bar{p}}$, which we shall define next. If $C$ is nonhyperelliptic, $\eta$ the square trivial line bundle corresponding to $\pi: \tilde{C} \rightarrow C$, (i.e. the unique line bundle on $C$ such that $\eta \neq \mathcal{O}_{C}, \pi^{*}(\eta)=\mathcal{O}_{\tilde{C}}$, for $\left.\pi^{*}: J(C) \rightarrow J(\tilde{C})\right)$, then the Prym canonical map $\varphi_{\eta}: C \rightarrow\left|\omega_{C} \otimes \eta\right|^{*}=\mathbb{P}\left(T_{0}(P)\right)$ is a morphism, and we denote by $\Lambda_{\bar{p}}$ the hyperplane in $\left|\omega_{C} \otimes \eta\right|$ parametrizing Prym canonical divisors on $C$ containing $\varphi_{\eta}(\bar{p})$. We denote by $\Gamma_{\bar{p}} \subset \Xi$ the Gauss divisor corresponding to $\Lambda_{\bar{p}}$, i.e. $\Gamma_{\bar{p}}=$ (the closure in $\Xi$ of) $\gamma_{\Xi}^{-1}\left(\Lambda_{\bar{p}}\right)$ where $\gamma_{\Xi}: \Xi_{\mathrm{sm}} \rightarrow\left|\mathcal{O}_{\Xi}(\Xi)\right|^{*} \cong \mathbb{P}\left(T_{0}(P)\right)^{*} \cong\left|\omega_{C} \otimes \eta\right|$ is the Gauss map defined on the smooth points of $\Xi$.

Equivalently, $\Gamma_{\bar{p}}$ is the Gauss divisor in $\left|\mathcal{O}_{\Xi}(\Xi)\right|$ corresponding to the point $\varphi_{\eta}(\bar{p})$ of the Prym canonical model $\varphi_{\eta}(C)$ of $C$ in $\left|\omega_{C} \otimes \eta\right|^{*}=\mathbb{P}\left(T_{0}(P)\right) \cong\left|\mathcal{O}_{\Xi}(\Xi)\right|$, where a direction $v$ in $T_{0}(P)$ corresponds to the divisor on $\Xi$ of the directional derivative $\partial_{v} \vartheta$, where $\vartheta$ is a theta function on $P$ vanishing simply on $\Xi$.

Theorem. Assuming the notation of the setup above, if $C$ is a smooth nonhyperelliptic curve of genus $g \geq 4, \pi: \tilde{C} \rightarrow C$ a connected étale double cover, $\bar{p}$ any point of $C$, and $\pi^{-1}(\bar{p})=\left\{p, p^{\prime}\right\}$, then

1) $\varphi\left(\mathcal{D}_{p} \cap \mathcal{D}_{p^{\prime}}\right)=\Gamma_{\bar{p}} \subset \Xi$, the Gauss divisor corresponding to the point $\varphi_{\eta}(\bar{p})$ on the Prym canonical model of $C$.

2) If furthermore $g \geq 5$ and $\bar{p}$ is a general point of $C$, then $\bar{p}$ is a ramification point of only finitely many $g_{4}^{1}$ 's, and for any such $\bar{p}, \Gamma_{\bar{p}}$ is normal and irreducible.

3) If a component of the branch divisor of the Gauss map were dual to the Prym canonical curve, then the divisors $\Gamma_{\bar{p}}$ would be singular in codimension one. 
Corollary. If $C$ is a smooth nonhyperelliptic curve of genus $g \geq 4, \pi: \tilde{C} \rightarrow C$ any connected étale double cover, and $B_{\gamma}$ is the (closure of the) branch divisor of the Prym Gauss map $\gamma: \Xi_{s m} \rightarrow\left|\mathcal{O}_{\Xi}(\Xi)\right|^{*} \cong\left|\omega_{C} \otimes \eta\right|$, then $B_{\gamma}$ does not contain the dual $\left(\varphi_{\eta}(C)\right)^{*}$ of the Prym canonical curve.

Proof of Corollary. It follows from the theorem, parts 1) and 2), that if $C$ has genus $g \geq 5$, and $\bar{p}$ is general on $C$, the Gauss divisor $\Gamma_{\bar{p}}$ is normal, hence nonsingular in codimension one, which contradicts the assumption that $B_{\gamma}$ contains $\varphi_{\eta}(C)^{*}$ by part 3). So assume $C$ has genus $g=4$. Then by Recillas' theorem [Re], the Prym variety $P(\tilde{C} / C)$ is a Jacobian $J(\Sigma)$ of a curve $\Sigma$ of genus 3 , hence by Andreotti's proof of Torelli $\left[\mathrm{A}\right.$, the branch divisor $B_{\gamma}$ is the union of the dual of the image in $\mathbb{P}^{2}$ of $\Sigma$ and the lines dual to the images of any ramification points of the canonical map $\varphi_{\omega}: \Sigma \rightarrow \mathbb{P}^{2}$. Since the Prym canonical map $\varphi_{\eta}$ on a nonhyperelliptic curve $C$ is a nonconstant morphism, the only way $B_{\gamma}$ can contain $\varphi_{\eta}(C)^{*}$ is if $\varphi_{\eta}(C)=\varphi_{\omega}(\Sigma)$. Now $\varphi_{\omega}(\Sigma)$ has degree either 4 or 2 and $\varphi_{\eta}(C)$ has degree a divisor of 6 . Thus we need only consider the case where the Prym canonical map $\varphi_{\eta}: C \rightarrow \Delta \subset \mathbb{P}^{2}$ is a degree three cover of a plane conic, i.e. is given by the composition of a $g_{3}^{1}$ and the quadratic Veronese map $\mathbb{P}^{1} \rightarrow \mathbb{P}^{2}$, which implies that $\omega_{C} \otimes \eta=\left(\varphi_{\eta}\right)^{*}(\mathcal{O}(1))=\mathcal{O}\left(2 \cdot g_{3}^{1}\right)$. But since $\Delta=\varphi_{\eta}(C)=\varphi_{\omega}(\Sigma), \Sigma$ is hyperelliptic of genus three, hence $P(\tilde{C} / C)=J(\Sigma)$ is a hyperelliptic Jacobian, so by [Mu1, Theorem, part (c), p.344], the genus four curve $C$ has an effective even theta characteristic. That implies $C$ has only one $g_{3}^{1}$, and for that one we have $\mathcal{O}\left(2 \cdot g_{3}^{1}\right)=\omega_{C}$, contradicting the fact deduced above that $\mathcal{O}\left(2 \cdot g_{3}^{1}\right)=\omega_{C} \otimes \eta$, since $\eta$ is nontrivial. This proves the corollary.

Proof of Theorem. Recall the conventions from the setup above; in particular, $g=$ $g(C), \tilde{g}=g(\tilde{C})=2 g-1, \operatorname{dim}(P)=\operatorname{dim}(X)=g-1, \operatorname{dim}(\Xi)=g-2, \operatorname{dim}\left(\Gamma_{\bar{p}}\right)=g-3$.

Steps for the proof of part 1). Assume $C$ is a smooth nonhyperelliptic curve of genus $g \geq 4, \pi: \tilde{C} \rightarrow C$ a connected étale double cover, $\bar{p}$ any point of $C$, $\pi^{-1}(\bar{p})=\left\{p, p^{\prime}\right\}$, and $\xi$ is the cohomology class of the Prym theta divisor $\Xi$. Then

i) the $(g-3)$ (i.e. highest) dimensional part of the subset $\varphi\left(\mathcal{D}_{p} \cap \mathcal{D}_{p^{\prime}}\right) \subset \Xi$ with its reduced scheme structure, has cohomology class $\xi^{2}$ in $H^{*}(P)$, (the class of a Gauss divisor on $\Xi$ ).

ii) The set $\varphi\left(\mathcal{D}_{p} \cap \mathcal{D}_{p^{\prime}}\right)$ is contained in the Gauss divisor $\Gamma_{\bar{p}}=$ the pullback of the hyperplane $\Lambda_{\bar{p}}$ corresponding to the Prym canonical point $\varphi_{\eta}(\bar{p})$.

iii) Corollary: $\varphi\left(\mathcal{D}_{p} \cap \mathcal{D}_{p^{\prime}}\right)=\Gamma_{\bar{p}}$, more precisely the set $\varphi\left(\mathcal{D}_{p} \cap \mathcal{D}_{p^{\prime}}\right)$ is the support of the reduced Cartier divisor $\Gamma_{\bar{p}}$ on $\Xi$.

Proof of i) (the cohomology class of the $(g-3)$ dimensional components of $\varphi\left(\mathcal{D}_{p} \cap \mathcal{D}_{p^{\prime}}\right)$. We will identify $\varphi\left(\mathcal{D}_{p} \cap \mathcal{D}_{p^{\prime}}\right)$ as a translate of one component of the "special subvariety" associated in $\left[\mathrm{B}\right.$ to the linear series $\left|\omega_{C}-2 \bar{p}\right|$. If $\bar{p}$ is any point of $C$, since $C$ is nonhyperelliptic, the system $|\omega-2 \bar{p}|$ has at most a base divisor of degree one, hence contains a reduced divisor. [Indeed, if $\bar{q}$ is a base point of $|\omega-2 \bar{p}|$, then $h^{0}(\omega-2 \bar{p}-\bar{q})=h^{0}(\omega-2 \bar{p})$, hence by RiemannRoch $h^{0}(2 \bar{p}+\bar{q})>h^{0}(2 \bar{p})=1$, so $2 \bar{p}+\bar{q}$ defines a $g_{3}^{1}$ on $C$. Then for any point $\bar{r}$ of $C, h^{0}(2 \bar{p}+\bar{q}+\bar{r})$ cannot equal 3 by Clifford's theorem (since $C$ is nonhyperelliptic and of genus $g \geq 4)$, so $h^{0}(2 \bar{p}+\bar{q}+\bar{r})=h^{0}(2 \bar{p}+\bar{q})=2$, hence by Riemann-Roch $h^{0}(\omega-2 \bar{p}-\bar{q}-\bar{r})<h^{0}(\omega-2 \bar{p}-\bar{q})$, i.e. the base divisor of $|\omega-2 \bar{p}|$ is only the single point $\bar{q}$ with multiplicity 1.] Thus the hypotheses of $[\mathrm{B}$, p.359] hold and $\pi^{-1}\left(\left|\omega_{C}-2 \bar{p}\right|\right) \subset \tilde{C}^{(2 g-4)}$ consists of two connected reduced components, 
$S_{0} \cup S_{1}$. We index them so that $S_{0}+p+p^{\prime} \subset X$, and $S_{1}+p+p^{\prime} \subset X^{-}$. By B, Remarque 1, p.360], $S_{0}$ has at most two types of irreducible components, those collapsing by $\tilde{\alpha}$ to lower dimensional subvarieties, and those on which $\tilde{\alpha}$ is birational. The same argument, i.e. the fact that $|D| \cong \mathbb{P}^{0}$ for a general point $D$ on a component of $S_{0}$ on which $\tilde{\alpha}$ is birational, proves that the image cycle $\tilde{\alpha}_{*}\left[S_{0}\right]$ is reduced, and is the component in the expected dimension $(g-3)$, of the cycle of the reduced image variety $V_{0}=\tilde{\alpha}\left(S_{0}\right)$, in Beauville's notation. Moreover, since $\operatorname{deg}\left(\omega_{C}-2 \bar{p}\right)=2 g-4>2\left(\operatorname{dim}\left|\omega_{C}-2 \bar{p}\right|\right)=2(g-3),[$ B, Thm.1, p.364] shows that the cohomology class of (the $g-3$ dimensional part of) $V_{0}$ in $P$ (after appropriate translation), is $\xi^{2}$. Since $\mathcal{D}_{p} \cap \mathcal{D}_{p^{\prime}}$ is the set of those precanonical divisors on $\tilde{C}$ which contain the points $p$ and $p^{\prime}$, it follows that $\mathcal{D}_{p} \cap \mathcal{D}_{p^{\prime}}=p+p^{\prime}+S_{0}$ as sets, and we give $\mathcal{D}_{p} \cap \mathcal{D}_{p^{\prime}}$ the scheme structure induced from $p+p^{\prime}+S_{0}$ as well, which is thus reduced. In particular, $\left.\operatorname{dim}\left(\mathcal{D}_{p} \cap \mathcal{D}_{p^{\prime}}\right)=\operatorname{dim}\left(S_{0}\right)=\operatorname{dim}\left(\mid \omega_{C}-2 \bar{p}\right) \mid\right)=g-3$. (In fact the natural intersection scheme structure for $\mathcal{D}_{p} \cap \mathcal{D}_{p^{\prime}}$ is also reduced, but we do not need this.) Then the $g-3$ dimensional part of $\varphi\left(\mathcal{D}_{p} \cap \mathcal{D}_{p^{\prime}}\right)=\tilde{\alpha}(p)+\tilde{\alpha}\left(p^{\prime}\right)+V_{0}$ also has cohomology class $\xi^{2}$ in $P$. QED for i).

Remark. In particular, since the class $\xi^{2}$ is nonzero, $\varphi$ is birational on at least one component of $\mathcal{D}_{p} \cap \mathcal{D}_{p^{\prime}}$.

Proof of ii), the inclusion relation $\varphi\left(\mathcal{D}_{p} \cap \mathcal{D}_{p^{\prime}}\right) \subset \Gamma_{\bar{p}}$. Since $C$ is nonhyperelliptic, the linear system $\left|\omega_{C} \otimes \eta\right|$ is base point free by Riemann Roch, and the Prym canonical map $\varphi_{\eta}$ is a morphism from $C$ to $\left|\omega_{C} \otimes \eta\right|^{*}$. We will examine the relation between the Prym canonical map of $C$, and the canonical maps of $C$ and $\tilde{C}$, within the common projective space $|\tilde{\omega}|^{*}=\mathbb{P} T_{0} \tilde{J}$. The linear space of differentials on $\tilde{C}$ decomposes as a direct sum of symmetric differentials and skew symmetric ones, corresponding respectively to the pullbacks of usual differentials on $C$ and of Prym differentials on $C$. Hence these define complementary subspaces of linear forms on $|\tilde{\omega}|^{*} \cong \mathbb{P}^{2 g-2}$ whose zero loci define disjoint subspaces of $|\tilde{\omega}|^{*}$ corresponding respectively to the Prym canonical space $\left|\omega_{C} \otimes \eta\right|^{*} \cong \mathbb{P}^{g-2}$ and to the canonical space $|\omega|^{*} \cong \mathbb{P}^{g-1}$ for $C$. Since $C$ and $\tilde{C}$ are nonhyperelliptic, they are embedded canonically in $|\omega|^{*}$ and $|\tilde{\omega}|^{*}$ respectively. Given a point $p$ on $\tilde{C}$, with corresponding point $\bar{p}=\pi(p)$ on $C$, the Prym canonical point $\varphi_{\eta}(\bar{p})$ in $\left|\omega_{C} \otimes \eta\right|^{*}$ is obtained by projecting $p$ into $\left|\omega_{C} \otimes \eta\right|^{*}$ from the center $|\omega|^{*}$. Since $|\omega|^{*}$ is the zero locus of the skew symmetric Prym differentials, which have no base locus on $\tilde{C}$ for $C$ nonhyperelliptic, $p$ does not lie in the center of projection. Thus the join $\left\langle p,|\omega|^{*}\right\rangle$ is one dimension larger than $|\omega|^{*}$ and the Prym canonical image $\varphi_{\eta}(\bar{p})$ equals the one point intersection $\left\langle p,|\omega|^{*}\right\rangle \cap\left|\omega_{C} \otimes \eta\right|^{*}$.

Claim 1 (cf. [T1, p.957, line 11]). If $L_{p, p^{\prime}}$ is the line in $|\tilde{\omega}|^{*}$ joining $p$ to $p^{\prime}$, the Prym canonical point $\varphi_{\eta}(\bar{p})=L_{p, p^{\prime}} \cap\left|\omega_{C} \otimes \eta\right|^{*}$.

Proof. Since the join $\left\langle p,|\omega|^{*}\right\rangle$ is defined by the skew symmetric differentials vanishing on $p$, and since a skew symmetric differential vanishes at $p$ if and only if it vanishes at $p^{\prime}$, the three joins $\left\langle p,|\omega|^{*}\right\rangle,\left\langle p^{\prime},|\omega|^{*}\right\rangle$, and $\left\langle p, p^{\prime},|\omega|^{*}\right\rangle$ are all equal to each other, and thus $\varphi_{\eta}(\bar{p})$ also equals the intersection $\left\langle p, p^{\prime},|\omega|^{*}\right\rangle \cap\left|\omega_{C} \otimes \eta\right|^{*}$. Since we have the inclusion $L_{p, p^{\prime}} \subset\left\langle p, p^{\prime},|\omega|^{*}\right\rangle$, we also have an inclusion $L_{p, p^{\prime}} \cap\left|\omega_{C} \otimes \eta\right|^{*} \subset$ $\left\langle p, p^{\prime},|\omega|^{*}\right\rangle \cap\left|\omega_{C} \otimes \eta\right|^{*}=\left\{\varphi_{\eta}(\bar{p})\right\}$. A symmetric differential also vanishes at $p$ if and only if it vanishes at $p^{\prime}$, so the three joins $\left\langle p,|\omega \otimes \eta|^{*}\right\rangle,\left\langle p^{\prime},|\omega \otimes \eta|^{*}\right\rangle$, and $\left\langle p, p^{\prime},|\omega \otimes \eta|^{*}\right\rangle$ are also all equal to each other. Since the join $\left\langle p, p^{\prime},|\omega \otimes \eta|^{*}\right\rangle$ is thus 
at most one dimension larger than the space $|\omega \otimes \eta|^{*}$, the intersection $L_{p, p^{\prime}} \cap\left|\omega_{C} \otimes \eta\right|^{*}$ is nonempty and hence equals $\left\{\varphi_{\eta}(\bar{p})\right\}$. QED Claim 1 .

Claim 2. If $\gamma_{\Xi}$ is the Gauss morphism defined on the smooth points $\Xi_{\mathrm{sm}} \subset \Xi$, then $\varphi\left(\mathcal{D}_{p} \cap \mathcal{D}_{p^{\prime}}\right) \cap \Xi_{\mathrm{sm}} \subset \gamma_{\Xi}^{-1}\left(\Lambda_{\bar{p}}\right)$, where $\Lambda_{\bar{p}}$ is the hyperplane in $\left|\omega_{C} \otimes \eta\right|$ of Prym canonical divisors which contain $\bar{p}$.

Proof. We must show, for $x$ in $\mathcal{D}_{p} \cap \mathcal{D}_{p^{\prime}}$, if $\varphi(x)$ lies in $\Xi_{\mathrm{sm}}$, then the hyperplane $\gamma_{\Xi}(\varphi(x))$ contains $\varphi_{\eta}(\bar{p})$. We know that if $D_{x}$ is the canonical divisor on $\tilde{C}$ corresponding to the point $x$, then the linear span $\bar{D}_{x}$ of this divisor in $|\tilde{\omega}|^{*}$ contains $p$ and $p^{\prime}$ hence also the line $L_{p, p^{\prime}}$. Moreover, the span $\bar{D}_{x}$ is one of the rulings on the quadric tangent cone $\tilde{Q}_{\varphi(x)}$ to $\tilde{\Theta}$ at the point $\varphi(x)$. Thus we have inclusions $\varphi_{\eta}(\bar{p})=L_{p, p^{\prime}} \cap|\omega \otimes \eta|^{*} \subset \bar{D}_{x} \cap|\omega \otimes \eta|^{*} \subset \tilde{Q}_{\varphi(x)} \cap|\omega \otimes \eta|^{*}$. However, by Mu1. p.343] this last intersection is set theoretically just the tangent space to $\Xi$ at $\varphi(x)$, i.e. is equal to $\gamma_{\Xi}(\varphi(x))$. QED Claim 2.

Corollary. $\varphi\left(\mathcal{D}_{p} \cap \mathcal{D}_{p^{\prime}}\right) \subset \Gamma_{\bar{p}}$.

Proof. Since $\Gamma_{\bar{p}}$ is defined as the closure of $\gamma_{\Xi}^{-1}\left(\Lambda_{\bar{p}}\right)$ in $\Xi$, Claim 2 implies that $\varphi\left(\mathcal{D}_{p} \cap \mathcal{D}_{p^{\prime}}\right) \cap \Xi_{\mathrm{sm}} \subset \Gamma_{\bar{p}}$. To see that $\varphi\left(\mathcal{D}_{p} \cap \mathcal{D}_{p^{\prime}}\right) \cap \operatorname{sing} \Xi \subset \Gamma_{\bar{p}}$ also, we recall that sing $\Xi$ is contained in every Gauss divisor on $\Xi$, hence, in particular $\varphi\left(\mathcal{D}_{p} \cap \mathcal{D}_{p^{\prime}}\right) \cap \operatorname{sing} \Xi \subset \operatorname{sing} \Xi \subset \Gamma_{\bar{p}}$. I.e. recall the global sections of $\mathcal{O}_{\Xi}(\Xi)$ on $\Xi$, are spanned by the partial derivatives $\partial \vartheta / \partial z_{j}$ of a theta function for $\Xi$, (in terms of linear coordinates $z_{j}$ on the universal cover of $\mathrm{P}$ ), hence all Gauss divisors contain the base locus sing $\Xi$ of the partial derivatives. QED for Cor. and for ii).

We pause for some interesting remarks before resuming the argument with the proof of iii) below.

Remark. This argument shows that for any point $\varphi(x)$ of $\Xi$, smooth or not, if $\mathbb{P T C}_{\varphi(x)}(\tilde{\Theta})$ denotes the projectivized tangent cone to $\tilde{\Theta}$ at $\varphi(x)$ (translated to a hypersurface in $\left.\mathbb{P} T_{0} \tilde{J} \cong|\tilde{\omega}|^{*}\right)$, then the intersection $\mathbb{P} T C_{\varphi(x)}(\tilde{\Theta}) \cap|\omega \otimes \eta|^{*}$ contains the Prym canonical point $\varphi_{\eta}(\bar{p})$ as long as $\mathcal{D}_{p} \cap \mathcal{D}_{p^{\prime}}$ intersects the fiber $\varphi^{-1}(\varphi(x))$ nontrivially. Since to meet $\mathcal{D}_{p} \cap \mathcal{D}_{p^{\prime}}$ imposes at most two conditions on the fiber $\varphi^{-1}(\varphi(x))$, any fiber $\varphi^{-1}(\varphi(x))$ of dimension $\geq 2$ meets $\mathcal{D}_{p} \cap \mathcal{D}_{p^{\prime}}$ for every point $p$. Hence for any point $\varphi(x)$ of multiplicity $\geq 3$ on $\tilde{\Theta}$, the Prym canonical curve $\varphi_{\eta}(C)$ is contained in the intersection $\mathbb{P} T C_{\varphi(x)}(\tilde{\Theta}) \cap|\omega \otimes \eta|^{*}$. When, furthermore, $\operatorname{PTC}_{\varphi(x)}(\tilde{\Theta})$ does not contain the projective tangent space $|\omega \otimes \eta|^{*}$ to the Prym variety, then the tangent cone to $\Xi$ at $\varphi(x)$ equals the intersection $\mathbb{P T C}_{\varphi(x)}(\tilde{\Theta}) \cap$ $|\omega \otimes \eta|^{*}$ (as subsets of the Prym canonical space $|\omega \otimes \eta|^{*} \cong \mathbb{P}^{g-2}$ ) and thus contains the Prym canonical curve. In particular, this occurs at so called "stable" double points as we will observe next.

Definition [T1, p.960]. Given a double cover $\tilde{C} / C$ and associated Prym variety, a point of $\Xi(\tilde{C} / C)$ which is of even multiplicity 4 or more on $\tilde{\Theta}$, is called a "stable" singularity of the Prym theta divisor.

Remarks. 1) The property of being "stable" is not intrinsic to the geometry of $\Xi$, but depends on the particular double cover $\tilde{C} / C$ giving rise to $\Xi$ [D2, p.546].

2) Stable singularities were originally introduced simply as "case 2" singularities in [Mu1, p.345]. 
Corollary [T1. Lemma 2.3, p.963]. The tangent cone to $\Xi(\tilde{C} / C)$ at a stable double point contains the Prym canonical model of $C$.

Proof. If $\varphi(x)$ is a stable singularity such that the intersection $\mathbb{P} T C_{\varphi(x)}(\tilde{\Theta}) \cap|\omega \otimes \eta|^{*}$ contains the space $|\omega \otimes \eta|^{*}$, then the restriction to $\Xi$ of an equation for $\tilde{\Theta}$ would begin with a term of degree at least 6 , hence $\varphi(x)$ would be a point of multiplicity $\geq 3$ on $\Xi$. Hence at a stable double point the intersection $\mathbb{P} T C_{\varphi(x)}(\tilde{\Theta}) \cap|\omega \otimes \eta|^{*}$ is proper, and the result follows from the remarks just above the previous definition. QED Corollary.

Remarks. 1) The statements of Claim 1 above and of the previous corollary appear in [T1 as cited, but because the "Correction" [T2] to parts of that paper has appeared, we include proofs of the relevant statements for completeness.

2) It is an open problem to determine precisely those cases when the intersection of the tangent quadrics to $\Xi$ at all stable double points of $\Xi$ equals precisely the Prym canonical curve. Debarre [D1] proved this is true for a general Prym variety of dimension $\geq 8$, but it fails for general Prym varieties of dimension $\leq 6$.

Now we resume the argument.

Proof of iii), $\varphi\left(\mathcal{D}_{p} \cap \mathcal{D}_{p^{\prime}}\right)=\Gamma_{\bar{p}}$. So far we know the following: $\varphi\left(\mathcal{D}_{p} \cap \mathcal{D}_{p^{\prime}}\right)=W_{1} \cup$ $W_{2}$, where $W_{1}$ is a reduced pure $(g-3)$ dimensional (i.e. pure codimension one) closed algebraic subset of $\Xi \subset P, W_{2}$ is a (possibly empty) closed algebraic set of dimension less than $(g-3)$, and $\varphi\left(\mathcal{D}_{p} \cap \mathcal{D}_{p^{\prime}}\right) \subset \Gamma_{\bar{p}}$, where $\Gamma_{\bar{p}} \subset \Xi$ is a Gauss divisor, i.e. an effective Cartier divisor in the linear system $\left|\mathcal{O}_{\Xi}(\Xi)\right|$ on $\Xi$, and the two divisors $W_{1}$ and $\Gamma_{\bar{p}}$ have the same homology class in $H_{*}(P, \mathbb{Z})$ by i). In other words, we have an inequality $W_{1} \leq \Gamma_{\bar{p}}$ of Weil divisors on $\Xi$, and $W_{1}$ and $\Gamma_{\bar{p}}$ have the same homology class on $P$. Then we can immediately conclude that $W_{1}=\Gamma_{\bar{p}}$ by the following lemma.

Lemma 1. Suppose that we have an inequality $D_{1} \leq D_{2}$ of algebraic $k$-cycles on a projective variety $V$, and that $D_{1}$ and $D_{2}$ have the same homology class in $H_{*}(V, \mathbb{Z})$. Then $D_{1}=D_{2}$.

Proof. By embedding $V$ in $\mathbb{P}^{N}$, we may assume that $D_{1}$ and $D_{2}$ are algebraic $k$ cycles in $\mathbb{P}^{N}$ satisfying $D_{2}=D_{1}+D^{\prime}$, where $D^{\prime}$ is an effective algebraic $k$-cycle in $\mathbb{P}^{N}$ with homology class 0 in $H_{2 k}\left(\mathbb{P}^{N}, \mathbb{Z}\right)$. Then $D^{\prime}$ must be the zero $k$-cycle. Otherwise, write $D^{\prime}=\Sigma n_{\alpha} W_{\alpha}$ (summation over a nonempty finite index set $\{\alpha\}$ ) with $n_{\alpha}>0$ and $W_{\alpha}$ (nonempty, irreducible) of dimension $k$ in $\mathbb{P}^{N}$. Then the intersection number of a codimension $k$-linear subspace of $\mathbb{P}^{N}$ with the homology class of $D^{\prime}$ is $\Sigma n_{\alpha} d_{\alpha}$, where $d_{\alpha}$ is the degree of $W_{\alpha}$ in $\mathbb{P}^{N}$; but $d_{\alpha}>0$, so $\Sigma n_{\alpha} d_{\alpha}>0$, and the homology class of $D^{\prime}$ is nonzero, contradiction. QED Lemma 1.

Now iii) follows since $W_{1}=\Gamma_{\bar{p}}$ by Lemma 1 , and $\varphi\left(\mathcal{D}_{p} \cap \mathcal{D}_{p^{\prime}}\right) \subset \Gamma_{\bar{p}}$ by ii), hence as sets $\varphi\left(\mathcal{D}_{p} \cap \mathcal{D}_{p^{\prime}}\right) \subset \Gamma_{\bar{p}}=W_{1} \subset W_{1} \cup W_{2}=\varphi\left(\mathcal{D}_{p} \cap \mathcal{D}_{p^{\prime}}\right)$, thus $\varphi\left(\mathcal{D}_{p} \cap \mathcal{D}_{p^{\prime}}\right)=\Gamma_{\bar{p}}$ as sets. This proves part 1) of the theorem.

Proof of Theorem, part 2): Normality of $\Gamma_{\bar{p}}=\varphi\left(\mathcal{D}_{p} \cap \mathcal{D}_{p^{\prime}}\right)$. First we prove $\mathcal{D}_{p} \cap \mathcal{D}_{p^{\prime}}$ is normal and irreducible.

Lemma 2. If $C$ is nonhyperelliptic of genus $g \geq 5$, and $\bar{p}$ is a ramification point of only a finite number of $g_{4}^{1}$ 's on $C$, then $\left(\mathcal{D}_{p} \cap \mathcal{D}_{p^{\prime}}\right)$ is normal and irreducible. 
Proof. Since $\mathcal{D}_{p} \cap \mathcal{D}_{p^{\prime}}=p+p^{\prime}+S_{0}$, it suffices to prove $S_{0}$ is normal and irreducible. Thus it suffices to verify the hypotheses of [B Cor., Prop. 3, p.365], i.e. that the linear system $\left|\omega_{C}(-2 \bar{p})\right|$ is base point free and defines a birational morphism with at most one ramification point in each fiber, and no ramification point of index $\geq 4$. Our hypothesis implies that $\bar{p}$ is not a ramification point of any $g_{3}^{1}$ on $C$, hence for every point $\bar{q}, h^{0}(2 \bar{p}+\bar{q})=1=h^{0}(2 \bar{p})$, so $h^{0}\left(\omega_{C}(-2 \bar{p})\right)>h^{0}\left(\omega_{C}(-2 \bar{p}-\bar{q})\right)$, and the system $\left|\omega_{C}(-2 \bar{p})\right|$ is base point free. In order for a fiber of the morphism associated to $\left|\omega_{C}(-2 \bar{p})\right|$ to dominate a divisor $\bar{q}+\bar{r}$, we must have $h^{0}(2 \bar{p}+\bar{q}+\bar{r})=2$, so that $2 \bar{p}+\bar{q}+\bar{r}$ is one of the finitely many divisors of $g_{4}^{1}$ 's which dominate $2 \bar{p}$. Hence for every point $\bar{q}$ not in the support of one of these divisors, $\bar{q}$ is a nonsingular, singleton fiber of the morphism, which is thus birational onto its image. On the other hand, if $\bar{q}+\bar{r}+\bar{s}+\bar{t}$ is any divisor of degree 4 which is dominated by the fiber containing $\bar{q}$, then we must have $h^{0}\left(\omega_{C}(-2 \bar{p}-\bar{q})\right)=h^{0}\left(\omega_{C}(-2 \bar{p}-\bar{q}-\bar{r}-\bar{s}-\bar{t})\right)$, hence $h^{0}(2 \bar{p}+\bar{q}+\bar{r}+\bar{s}+\bar{t})=4$. Since the linear system $|2 \bar{p}+\bar{q}+\bar{r}+\bar{s}+\bar{t}|$ is thus a $g_{6}^{3}$, and $C$ is nonhyperelliptic with $g(C) \geq 5$, this contradicts Clifford's theorem. Thus there cannot be more than one ramification point in each fiber, and no ramification point can have index $\geq 4$. QED Lemma 2 .

Next we check that the hypotheses of Lemma 2 hold at a general point of the curves under consideration.

Lemma 3. If $C$ is nonhyperelliptic of genus $g \geq 5$, then at most a finite number of points $\bar{p}$ of $C$ are ramification points of infinitely many $g_{4}^{1}$ 's.

Proof. By Martens' theorem, [ACGH, p.191], the variety $W_{4}^{1} \subset \operatorname{Pic}^{4}(C)$ parametrizing all line bundles $L$ defining $g_{4}^{1}$ 's on $C$ is of dimension $\leq 1$. Consider the incidence variety $I \subset C \times W_{4}^{1}$ consisting of pairs $(\bar{p}, L)$ such that $\bar{p}$ is a ramification point of $L$, and consider the projection maps $I \rightarrow W_{4}^{1}$ and $I \rightarrow C$. Since each $g_{4}^{1}$ has at most a finite number of ramification points the map $I \rightarrow W_{4}^{1}$ is finite, so $\operatorname{dim}(I) \leq 1$. Then the map $I \rightarrow C$ has infinite fibers over at most a finite number of points of $C$, i.e. at most a finite number of points of $C$ are ramification points of infinitely many $g_{4}^{1}$ 's, as claimed. QED Lemma 3.

Next we prove normality and irreducibility of the image $\varphi\left(\mathcal{D}_{p} \cap \mathcal{D}_{p^{\prime}}\right)$.

Lemma 4. If $C$ is nonhyperelliptic, $g(C) \geq 5$, and $\left(\mathcal{D}_{p} \cap \mathcal{D}_{p^{\prime}}\right)$ is normal and irreducible, then $\varphi\left(\mathcal{D}_{p} \cap \mathcal{D}_{p^{\prime}}\right)=\Gamma_{\bar{p}} \subset \Xi$ is also normal and irreducible.

Proof. $\varphi\left(\mathcal{D}_{p} \cap \mathcal{D}_{p^{\prime}}\right)$ is irreducible since $\mathcal{D}_{p} \cap \mathcal{D}_{p^{\prime}}$ is. For normality, we first show $\varphi$ is an injective immersion except on a subset of $\mathcal{D}_{p} \cap \mathcal{D}_{p^{\prime}}$ whose image has codimension at least 2 in $\varphi\left(\mathcal{D}_{p} \cap \mathcal{D}_{p^{\prime}}\right)$. Consider the maps $\varphi: \mathcal{D}_{p} \rightarrow \Xi$, and $\varphi:\left(\mathcal{D}_{p} \cap \mathcal{D}_{p^{\prime}}\right) \rightarrow \Gamma_{\bar{p}} \subset$ $\Xi$. First throw out the singular points $Z_{1}=\operatorname{sing}(\Xi)$, from $\Xi$, and throw out also their inverse images $\varphi^{-1}\left(Z_{1}\right)=W_{1}$ from $\mathcal{D}_{p} \cap \mathcal{D}_{p^{\prime}}$. Since $\Xi$ is the Prym theta divisor of a connected étale double cover of smooth curves, and $C$ is nonhyperelliptic of genus $g \geq 5$, Mumford's theorem [Mu1, p.344, part d] implies $Z_{1}$ has codimension $\geq 3$ in $\Xi$, hence $\varphi\left(W_{1}\right) \subset Z_{1}$ has codimension $\geq 2$ in the divisor $\Gamma_{\bar{p}} \subset \Xi$. Then for all $D$ in $\left(\mathcal{D}_{p} \cap \mathcal{D}_{p^{\prime}}\right)-W_{1}, \varphi(D)$ is a smooth point of $\Xi$, and $\varphi: X \rightarrow \Xi$ is a $\mathbb{P}^{1}$ bundle over a neighborhood of $\varphi(D)$. Then we we throw out of $\Xi$ also the set $Z_{2}$ of divisor classes with $p$ and $p^{\prime}$ both as base points, and throw out from $\mathcal{D}_{p} \cap \mathcal{D}_{p^{\prime}}$ the inverse image set $\varphi^{-1}\left(Z_{2}\right)=W_{2}$. Since $\varphi: W_{2} \rightarrow \varphi\left(W_{2}\right)=Z_{2}$ has positive dimensional fibers, and $\varphi: \mathcal{D}_{p} \cap \mathcal{D}_{p^{\prime}} \rightarrow \Gamma_{\bar{p}}$ is birational by the remark following the proof of Step (i), part 1 of the theorem, $W_{2} \subset\left(\mathcal{D}_{p} \cap \mathcal{D}_{p^{\prime}}\right)$ is a proper exceptional 
subvariety of codimension $\geq 1$, and hence $\varphi\left(W_{2}\right) \subset \Gamma_{\bar{p}}$ has codimension $\geq 2$. Then for $D$ in $\left(\mathcal{D}_{p} \cap \mathcal{D}_{p^{\prime}}\right)-W_{1}-W_{2}$, either $p$ or $p^{\prime}$ is not a base point for the pencil $|D|$, hence either the map $\varphi: \mathcal{D}_{p} \rightarrow \Xi$ or the map $\varphi: \mathcal{D}_{p^{\prime}} \rightarrow \Xi$ is, near $D$, a bijective map to a smooth variety, hence a local isomorphism by Zariski's Main Theorem. Thus, near such a point $D$ the map $\varphi: \mathcal{D}_{p} \cap \mathcal{D}_{p^{\prime}} \rightarrow \Gamma_{\bar{p}}$ is the restriction of a local isomorphism, hence $\varphi$ is immersive on $\left(\mathcal{D}_{p} \cap \mathcal{D}_{p^{\prime}}\right)-W_{1}-W_{2}$. Since the fibers of $\varphi: \mathcal{D}_{p} \cap \mathcal{D}_{p^{\prime}} \rightarrow \Xi$ are projective spaces, $\varphi$ immersive implies $\varphi$ is also injective on $\left(\mathcal{D}_{p} \cap \mathcal{D}_{p^{\prime}}\right)-W_{1}-W_{2}$. Thus $\varphi: \mathcal{D}_{p} \cap \mathcal{D}_{p^{\prime}} \rightarrow \Gamma_{\bar{p}}$ is an injective immersion on the open set $\left(\mathcal{D}_{p} \cap \mathcal{D}_{p^{\prime}}\right)-W_{1}-W_{2}$, and the image $\varphi\left(W_{1} \cup W_{2}\right)$ of the complement has codimension at least 2 in $\varphi\left(\mathcal{D}_{p} \cap \mathcal{D}_{p^{\prime}}\right)=\Gamma_{\bar{p}}$. Then we throw out the subset $W_{3}=\operatorname{sing}\left(\mathcal{D}_{p} \cap \mathcal{D}_{p^{\prime}}\right) \subset\left(\mathcal{D}_{p} \cap \mathcal{D}_{p^{\prime}}\right)$. Since $\left(\mathcal{D}_{p} \cap \mathcal{D}_{p^{\prime}}\right)$ is assumed normal, $W_{3}$ has codimension at least 2 in $\left(\mathcal{D}_{p} \cap \mathcal{D}_{p^{\prime}}\right)$, hence $\varphi\left(W_{3}\right)$ has codimension at least 2 in $\Gamma_{\bar{p}}$. Then $\left(\mathcal{D}_{p} \cap \mathcal{D}_{p^{\prime}}\right)-W_{1}-W_{2}-W_{3}$ is smooth and maps isomorphically to its image by $\varphi$; consequently, the image of this set is a smooth subset of $\Gamma_{\bar{p}}$ whose complement has codimension at least 2. Since $\Gamma_{\bar{p}}$ is a divisor in the Gauss linear system, it is a Cartier divisor, hence a local complete intersection in $P$, and since it has been shown to be smooth in codimension one, it is normal. QED Lemma 4.

We have shown that if $\bar{p}$ is a general point of $C$ and $C$ is nonhyperelliptic of genus $g \geq 5$, then $\bar{p}$ is a ramification divisor of only finitely many $g_{4}^{1}$ 's on $C$, and that for any such $\bar{p}, \varphi\left(\mathcal{D}_{p} \cap \mathcal{D}_{p^{\prime}}\right)=\Gamma_{\bar{p}}$ is normal and irreducible.

This completes the proof of part 2) of the theorem.

Remarks. We can be more precise about when $\Gamma_{\bar{p}}$ is normal. If $C$ has only finitely many $g_{4}^{1}$ 's, then the previous lemmas imply $\Gamma_{\bar{p}}$ is normal for every $\bar{p}$ on $C$. In particular, by [Mu1, Theorem, p.348], if $g(C) \geq 6$ and $C$ is neither trigonal nor bi-elliptic (i.e. not a double cover of an elliptic curve) nor a plane quintic, then $C$ has only finitely many $g_{4}^{1}$ 's. If $C$ is a plane quintic, all $g_{4}^{1}$ 's are of form $\left|g_{5}^{2}-\bar{q}\right|$ for some point $\bar{q}$ on $C$. [Since the net of conics containing three noncollinear points of $\mathbb{P}^{2}$ have no other base point, a net of conics has four base points only if the points are collinear. By the adjunction formula and Riemann Roch, thus every divisor of a $g_{4}^{1}$ on a plane quintic consists of four collinear points.] Hence a point $\bar{p}$ can only be a ramification point of those series $\left|g_{5}^{2}-\bar{q}\right|$ such that the tangent line to $C$ at $\bar{p}$ contains $\bar{q}$, a finite set of $g_{4}^{1}$ 's, hence again $\Gamma_{\bar{p}}$ is normal for every point $\bar{p}$ of a plane quintic $C$. If $C$ is bi-elliptic of genus $g \geq 6$, then by $\mathrm{R}$ Prop. 2.5, Cor. 2.6, pp. 234-235], there is a unique double cover $C \rightarrow E$ of an elliptic curve $E$, and every $g_{4}^{1}$ on $C$ is pulled back from a $g_{2}^{1}$ on $E$, (cf. also [S, p.129]). Hence if $\bar{p}$ is not a ramification point of the double cover $C \rightarrow E$, then $\bar{p}$ is a ramification point of only finitely many $g_{4}^{1}$ 's, and hence $\Gamma_{\bar{p}}$ is normal. If $C$ is trigonal and has a base point free $g_{4}^{1}$, then the two series $g_{3}^{1}$ and $g_{4}^{1}$ give a birational map from $\mathrm{C}$ to a curve of type $(3,4)$ on $\mathbb{P}^{1} \times \mathbb{P}^{1}$, hence of arithmetic genus $\leq 6$. Hence if $C$ is trigonal of genus $g \geq 7$, then every $g_{4}^{1}$ is of form $\bar{q}+g_{3}^{1}$, for the unique $g_{3}^{1}$ on $C$, hence if $\bar{p}$ is not a ramification point of the unique $g_{3}^{1}$, then $\bar{p}$ is a ramification point of only the one $g_{4}^{1}=\bar{p}+g_{3}^{1}$. Hence $\Gamma_{\bar{p}}$ is normal if $\bar{p}$ is not a ramification point of the $g_{3}^{1}$. If $g(C)=4$, and $\Gamma_{\bar{p}}$ is singular, Welters' singularity criterion (cf. [B] Prop. 3 , p.365]) implies that $\omega_{C}=|2 \bar{p}+2 \bar{q}+2 \bar{r}|$ for some points $\bar{q}, \bar{r}$ on $C$, i.e. $\mathcal{O}(\bar{p}+\bar{q}+\bar{r})$ is an effective theta characteristic on $C$. Hence if $C$ has no effective even theta characteristic, and $\bar{p}$ is not in the support of any of the finitely many odd theta characteristics of $C$, then $\Gamma_{\bar{p}}$ is normal (i.e. a smooth curve). However, if $C$ has 
an effective even theta characteristic, there is a double cover for which the Prym variety is a hyperelliptic Jacobian, hence $\Xi$ is singular. Since all Gauss divisors on $\Xi$ contain the singular point, hence all Gauss divisors $\Gamma$ on $\Xi$, including $\Gamma_{\bar{p}}$ for every $\bar{p}$ on $C$, are singular. Since here every $\Gamma$ is a curve, all Gauss divisors are nonnormal.

Proof of 3), singularities of "branch" Gauss divisors. Let $(A, \Theta)$ be a $d$-dimensional p.p.a.v. with (reduced and) irreducible theta divisor, let $\Theta_{s m}$ be the smooth points of $\Theta$, and let $\gamma: \Theta_{s m} \rightarrow \mathbb{P}^{*} T_{0} A \cong\left(\mathbb{P}^{d-1}\right)^{*}$ be the Gauss map, i.e. the map such that $\gamma(p)=T_{p} \Theta$, translated to a hyperplane in $T_{0} A$. Since $\Theta$ is irreducible and ample, the Gauss map is generically finite and dominant [K] Cor. 9.11, p.85], hence the differential of $\gamma$ is generically invertible, and the locus where it is noninvertible is defined locally by the determinant of the differential, hence has pure codimension one in $\Theta_{s m}$. The ramification locus of $\gamma$ is the subset $R$ of $\Theta_{s m}$ where the differential of $\gamma$ is not invertible (as a linear map of $d-1$ dimensional linear spaces) and the branch divisor $B$ is the codimension one part of the closure of the image of $R$ in $\mathbb{P}^{*} T_{0} A$.

Next we observe that tangent hyperplanes to the branch divisor $B$ yield singular members in the Gauss linear system on $\Theta_{s m}$; (note: all Gauss divisors on $\Theta$ are singular at all points of $\operatorname{sing} \Theta)$.

Lemma 5. Let $S$ be an irreducible component of the branch divisor of $\gamma, y$ a smooth point of $S, H$ the tangent hyperplane to $S$ at $y$, and $D=\gamma^{-1}(H)$ the divisor in the Gauss linear system associated to $H$. If $x$ is a smooth point of $\Theta$ such that $\gamma(x)=y$ and the image of the tangent space $T_{x} \Theta$ under the differential $\gamma^{\prime}(x)$ is contained in (the tangent space at $y$ to) $H$, then $D$ is singular at $x$.

Proof. If $\lambda$ is a local equation for $H$ near $y$, and $(\lambda \circ \gamma)$ the composed local equation for $D$ near $x$, then the linear term of $(\lambda \circ \gamma)$ is zero at $x$ since it defines the linear functional $\lambda^{\prime}(y) \circ \gamma^{\prime}(x)$, which is zero by hypothesis. QED Lemma 5.

Proposition. Let $\gamma: \Theta_{s m} \rightarrow\left(\mathbb{P}^{d-1}\right)^{*}$ be the Gauss map on an irreducible theta divisor of a d-dimensional principally polarized abelian variety, $d \geq 3$. If $S \subset$ $\left(\mathbb{P}^{d-1}\right)^{*}$ is a component of the branch divisor of $\gamma$ whose dual variety $S^{*}$ is a curve, if $H$ is a tangent hyperplane to $S$ at a general point of $S$, and $D=\gamma^{-1}(H)$ is the corresponding Gauss divisor, then $D$ is singular in codimension one.

Proof. After resolving the map $\gamma$ to make it regular, let $R$ be the closure in the normalized graph of $\gamma$, of the set \{smooth points $x$ in $\Theta_{s m}$ at which $\gamma^{\prime}(x)$ is not invertible $\}$. There must be a component $R_{1}$ of $R$ such that $\gamma\left(R_{1}\right)=S$, and since $R_{1}$ and $S$ are irreducible of the same dimension, the induced map $\gamma_{1}: R_{1} \rightarrow S$ is generically finite and surjective. We remove from $S$ the images of all added points, i.e. of all points of $R_{1}-\Theta_{s m}$, and remove also from $S$ all singular points of $S$, and all images of singular points of $R_{1}$. We then remove from $R_{1}$ all preimages of points removed from $S$, leaving a surjective map $\gamma_{2}: U \rightarrow V$ from an open smooth subset $U \subset R_{1}$, onto an open smooth subset $V \subset S$. By [Mu2, p.42], every point $y$ of $V$ outside a proper Zariski closed subset has the property that at every point $x$ of $\gamma_{2}^{-1}(y)$ in $U$, the differential $\gamma_{2}^{\prime}(x)$ has image equal to the tangent space $T_{y} S$. Since for $x$ in $U \subset R, \operatorname{dim}_{y} S \geq \operatorname{rank}_{x} \gamma^{\prime}(x)$, it follows that the image of $\gamma^{\prime}(x)$ also lies in $T_{y} S$. If $H$ is the tangent hyperplane to $S$ at $y$, it follows that the hypotheses of the previous lemma hold and hence $D=\gamma^{-1}(H)$ is singular at $x$. Since we are 
assuming $S^{*}$ is a curve, for each given tangent hyperplane $H$ to $S$, the set of points $y$ of $S$ such that $H=T_{y} S$, has codimension one in $S$. Thus for a general $H$, the set of points $x$ at which $\gamma^{-1}(H)=D$ is singular, has codimension one in $D$. QED Proposition. This completes the proof of the theorem.

Remarks on the hyperelliptic case. Given a connected étale double cover $\pi: \tilde{C} \rightarrow$ $C$, where $g(C) \geq 4$ and $C$ is hyperelliptic but $\tilde{C}$ is nonhyperelliptic, the Prym variety $P(\tilde{C} / C)$ is a product of two hyperelliptic Jacobians [Mu1, p.346], hence $\Xi(\tilde{C} / C)$ is reducible, and the rational Gauss map $\gamma_{\Xi}$ is the disjoint union of the Gauss maps on the two components of $\Xi$. The two images lie in disjoint linear subspaces of $\mathbb{P} T_{0} P^{*}$, neither of which can contain the dual of the Prym canonical curve, since the Prym canonical map is a morphism to a spanning curve. If $\tilde{C}$ and $C$ are both hyperelliptic, then using [Mu1, p.346], the double cover $\pi$ corresponds to a square trivial line bundle $\eta=p-q$, where $p, q$ are Weierstrass points on $C$. Then the rational Prym canonical map $\varphi_{\eta}: C \rightarrow \mathbb{P}^{g-2}$ is not a morphism but has base divisor $p+q$, the Prym variety $P(\tilde{C} / C) \cong J(\Sigma)$ is isomorphic to the Jacobian of a hyperelliptic curve $\Sigma$ of genus $g-1$, and the image of the rational map $\varphi_{\eta}$ is the same as the image of the canonical map $\varphi_{\omega}: \Sigma \rightarrow \mathbb{P}^{g-2}$, i.e. is the rational normal curve in $\mathbb{P}^{g-2}$ of degree $g-2$. Consequently, by Andreotti's proof of Torelli for the hyperelliptic Jacobian $J(\Sigma)$, in this case alone the branch divisor of the Gauss map on $\Xi(\tilde{C} / C)=\Theta(\Sigma)$ contains the dual of the image curve $\varphi_{\eta}(C)$.

Remarks on the relation with Andreotti's result. There is one subtle difference between our result and an exact analog of Andreotti's: in his paper [A Andreotti works with the branch divisor of the normalized Nash blowup $N(\gamma)$ of the Gauss map $\gamma$ on a Jacobian theta divisor $\Theta$ [A, p.820], [ACGH, p.246], whereas we work with the branch divisor of the actual Gauss map on smooth points, as in GH. p.360] which could conceivably be smaller. I.e. Andreotti first extends the rational Gauss map to a morphism on the Nash blowup of $\Theta$, then takes the normalization, considers the locus of those points in the target over which the fiber is a finite set of exactly degree $(\gamma)$ distinct points, and finally takes the codimension one part of the complement of this locus as the branch divisor. Thus the branch divisor of $\gamma$ consists of the union of only those components of the branch divisor of $N(\gamma)$ which can be detected from the behavior of the derivative of $\gamma$ at smooth points of $\Theta$. For Prym varieties, we do not know at present whether the dual of the Prym canonical curve can occur as a component of the possibly larger branch divisor of $N(\gamma)$. However, for general Prym varieties of dimensions 4 and 5 , the theta divisor is smooth, the Gauss map is a morphism, so the Nash blowup is the identity map, and the two branch loci are the same. Hence our results do imply that for all classical Prym varieties of dimensions 4 and 5 with smooth theta divisors, the dual of the Prym canonical curve is not a component of the branch divisor of either $\gamma$ or $N(\gamma)$. As we have mentioned, for Jacobians the branch divisors of both $\gamma$ and $N(\gamma)$ are equal, but in some examples they can differ. In particular, Varley has shown (unpublished) that for the theta divisor of the 4 dimensional abelian variety studied in $[\mathrm{V}]$, the branch divisor of $\gamma$ is empty, while that of $N(\gamma)$ is the union of the quadrics dual to the tangent cones of $\Theta$ at the double points. The unpublished result of Adams, McCrory, Shifrin, and Varley, that for a general 4 dimensional principally polarized abelian variety, both the branch divisor of the Gauss map and its dual, are irreducible surfaces of degree 60 in $\mathbb{P}^{3}$, was mentioned in the talk AMSV1 and is based on results published in AMSV2. 
Open questions. For the theta divisor $\Theta$ of a Jacobian variety of a curve $C$ of genus $g$, precise information about the Gauss map follows from Riemann's theorem that $\Theta=\left\{\xi \in \operatorname{Pic}^{g-1}(C): h^{0}(\xi) \geq 1\right\}$ and the corollary that the Abel map $\alpha: C^{(g-1)} \rightarrow \Theta$ gives a birational resolution of $\Theta$. I.e. points $p$ of canonical space $\left|\omega_{C}\right|^{*}$ parametrize Gauss divisors on $\Theta$, where $p$ corresponds to the Abel image in $\Theta$ of those $D=x_{1}+\ldots+x_{g-1}$ in $C^{(g-1)}$ such that $p$ lies on a hyperplane containing $D$ in $\left|\omega_{C}\right|^{*}$. Of special interest are those Gauss divisors corresponding to points $p$ of the canonical model of $C$ in $\left|\omega_{C}\right|^{*}$, the only one-parameter family of reducible Gauss divisors on $\Theta(C)$ for $C$ nonhyperelliptic and $g \geq 4$. I.e. if $C^{(g-1)} \supset$ $\mathbb{D}_{p}=\left\{\right.$ those $D$ in $C^{(g-1)}$ which contain $\left.p\right\}$, then the Gauss divisor corresponding to $p$ is the reducible, hence nonnormal, hypersurface $\alpha\left(\mathbb{D}_{p}\right) \cup\left(\alpha\left(\mathbb{D}_{p}\right)\right)^{\prime}$, where $\left(\alpha\left(\mathbb{D}_{p}\right)\right)^{\prime}$ denotes the image of the set $\alpha\left(\mathbb{D}_{p}\right)$ under the involution $L \mapsto\left(\omega_{C}-L\right)$ on $\Theta$. (To see this is the only infinite family of reducible Gauss divisors on nonhyperelliptic Jacobian theta divisors for $g \geq 4$, note that if $p$ is a point of $\left|\omega_{C}\right|^{*}$ not on $C_{\omega}$, then after projection from $p$, the Gauss divisor defined by $p$ is parametrized by the family of those divisors of degree $g-1$ on the projected curve that lie in hyperplane sections, an irreducible family by the monodromy lemma p.111 of [ACGH], provided the projection is birational. If the projection is not birational and $g \geq 5$, it must be of degree 2 onto a curve of degree $g-1$ in $\mathbb{P}^{g-2}$, thus an elliptic curve, and there are only finitely many such bi-elliptic projections of $C$. Or if $g=4$, there can also be one projection of degree 3 onto a plane conic. Cf. ACGH, Exercise batch E, pp.2689].) Andreotti observed further that the canonical curve parametrizing reducible Gauss divisors is dual to the branch divisor of the Gauss map for a Jacobian. Hence the following questions arise for the Gauss map on the theta divisor of any indecomposable principally polarized abelian variety $(A, \Theta)$ :

i) Is there a nondegenerate (i.e. irreducible, spanning) curve of reducible Gauss divisors?

ii) Is there a nondegenerate curve of nonnormal Gauss divisors?

iii) Is the branch divisor dual to a nondegenerate curve?

For Jacobians we have seen that the canonical curve of Gauss divisors answers yes to each of these. Hence, if $g=\operatorname{dim}(A)$, we might even restrict the curve in these questions to be of genus $=g$, and degree $=2 g-2$.

We note that property i) implies ii) since Gauss divisors are connected on abelian varieties of dimension $\geq 3$, and iii) implies ii) by the previous proposition. It is natural to ask whether these properties characterize Jacobians. If $\mathcal{A}_{g}$ is the moduli space of p.p.a.v.'s $(A, \Theta)$ with $\operatorname{dim}(A)=g$, recall the Andreotti Mayer locus $\mathcal{A}_{g} \supset \mathcal{N}_{g-4}=\left\{\right.$ those $(A, \Theta)$ in $\mathcal{A}_{g}$ such that $\left.\operatorname{dim}(\sin g \Theta) \geq g-4\right\}$. Beauville and Debarre prove in BD1 that if $\left|\mathcal{O}_{\Theta}(\Theta)\right|$ contains even one reducible Gauss divisor, then $(A, \Theta)$ belongs either to $\mathcal{N}_{g-4}$ or to another subvariety $\mathcal{E}_{g}=\{(A, \Theta)$ such that there is an elliptic curve $E \subset A$ with $E \cdot \Theta=2\}$. In particular, the locus $\mathcal{J}_{g}$ of Jacobians is an irreducible component of those $(A, \Theta)$ such that $\left|\mathcal{O}_{\Theta}(\Theta)\right|$ contains a reducible Gauss divisor. They also give a way to produce, on certain p.p.a.v.'s isogenous to products (which constitute most known examples of p.p.a.v.'s in $\mathcal{N}_{g-4}$ ), degenerate families of reducible Gauss divisors, and conclude that the property of having a single reducible Gauss divisor seems not much stronger than that of belonging to $\mathcal{N}_{g-4}$. It is not apparent to us, however, that any of their examples has a nondegenerate curve of reducible Gauss divisors, hence property i) above may well be stronger, hence more characteristic of Jacobians. As to the existence of nonnormal Gauss divisors, the result stated in [BD2, Remarque 1, p.619] (that 
a p.p.a.v. with a nonnormal Gauss divisor is either isogenous to a product or belongs to $\mathcal{N}_{g-4}$ ) seems to imply also (since a general Jacobian is "simple") that Jacobians are an irreducible component of the set of those p.p.a.v.'s having a single nonnormal Gauss divisor. In particular, Jacobians appear to be a component of the set of p.p.a.v.'s satisfying any one of the three properties in questions i)-iii) above, but we do not know of any non-Jacobians satisfying any of them.

\section{REFERENCES}

[A] A. Andreotti, On a theorem of Torelli, Am. J. Math. 80 (1958), 801-828. MR 21:1309

[ACGH] E. Arbarello, M. Cornalba, P. Griffiths, and J. Harris, Geometry of Algebraic Curves, vol. I, Springer-Verlag, 1985. MR 86h:14019

[AMSV1] M. Adams, C. McCrory, T. Shifrin, and R. Varley, The Gauss map of a generic genus 4 theta divisor, Special session talk, A.M.S. meeting, Ohio State Univ., summer 1990.

[AMSV2] M. Adams, C. McCrory, T. Shifrin, and R. Varley, Invariants of Gauss maps of theta divisors, Proc. Sympos. Pure Math., vol. 54, Amer. Math. Soc., Providence, R.I., 1993, pp. 1-8. MR 94i:14034

[B] A. Beauville, Sous-variétés spéciales des variétés de Prym, Compositio Math. 45 (3) (1982), 357-383. MR 83f:14025

[BD1] A. Beauville and O. Debarre, Une relation entre deux approches du problème de Schottky, Invent. Math. 86 (1986), 195-205. MR 87k:14031

[BD2] A. Beauville and O. Debarre, Sur le problème de Schottky pour les variétés de Prym, Ann. Scuola Norm. Sup. Pisa, Serie IV, 14 (1987), 613-623. MR 89m:14014

[D1] O. Debarre, Sur le problème de Torelli pour les variétés de Prym, Am. J. of Math. 111 (1989), 111-134. MR 90b:14035

[D2] O. Debarre, Sur les variétés de Prym des courbes tétragonales, Ann. Scient. Éc. Norm. Sup. 4e serie, t. 21 (1988), 545-559. MR 90b:14034

[GH] P. Griffiths and J. Harris, Principles of Algebraic Geometry, John Wiley and Sons, 1978. MR 80b:14001

[K] G. Kempf, Complex Abelian Varieties and Theta Functions, Springer-Verlag, 1991. MR 92h:14028

[Mu1] D. Mumford, Prym Varieties I, Contributions to Analysis (Ahlfors, Kra, Maskit, Nirenberg, eds.), Academic Press, New York, 1974, pp. 325-350. MR 52:415

[Mu2] D. Mumford, Algebraic geometry I, Complex Projective Varieties, Grundlehren der Math. Wissenschaften 221, Springer-Verlag, 1976. MR 56:11992

[R] S. Ramanan, Ample divisors on abelian surfaces, Proc. London Math Soc. 51 (1985), 231-245. MR 87d:14034

[Re] S. Recillas, Jacobians of curves with a $g_{4}^{1}$ are Prym varieties of trigonal curves, Bol. Soc. Mat. Mex. 19 (1974), 9-13. MR 58:666

[S] V. V. Shokurov, Prym varieties: theory and applications, Math. USSR Izvestiya 23 (1) (1984), 83-147.

[T1] A. Tjurin, The geometry of the Poincaré theta divisor of a Prym variety, Math. USSR Izvestija 9 (5) (1975), 951-986.

[T2] A. Tjurin, Correction to the Paper "The geometry of the Poincaré theta divisor of a Prym variety", Math. USSR Izvestija 12 (2) (1978), 438.

[V] R. Varley, Weddle's surfaces, Humbert's curves, and a certain 4-dimensional abelian variety, Am. J. of Math. 108 (1986), 931-952. MR 87g:14050

[Ve] A. Verra, The degree of the Gauss map for a general Prym theta-divisor, preprint, Università di Roma 3, 25 pages, 1998.

[W] G. E. Welters, Abel-Jacobi isogenies for certain types of Fano threefolds, Mathematisch Centrum, Amsterdam, 1981. MR 84k:14035

Department of Mathematics, University of Georgia, Athens, Georgia 30602

E-mail address: roy@math.uga.edu

Department of Mathematics, University of Georgia, Athens, Georgia 30602

E-mail address: rvarley@math.uga.edu 